\title{
Políticas públicas de saúde voltadas à atenção à prostituta: breve resgate histórico
}

Priscila de Souza Aquino' ${ }^{1}$ Lorena Barbosa Ximenes²

Ana Karina Bezerra Pinheiro ${ }^{3}$

Estudo reflexivo acerca das políticas públicas de saúde da mulher, notadamente relativas às prostitutas. No início do século XX, a saúde da mulher foi incorporada às políticas nacionais, mas era limitada ao cuidado materno-infantil. Com a criação do PAISM, o foco direcionou-se à saúde da mulher. O Programa Nacional de DST/Aids foi criado para enfrentar a disseminação do HIV. A política de distribuição de preservativos promoveu maior autonomia feminina. O PNAISM surgiu para implementar os direitos humanos das mulheres. As questões de gênero, vulnerabilidade individual e educação aos pares proporcionaram maior visibilidade e poder de voz às categorias marginalizadas.

Descritores: Políticas públicas; Prostituição; Formulação de políticas.

\section{Public politics of health directed to the attention to the prostitute: brief historical rescue}

Reflective study about the public politics for women's health, especially concerning the prostitutes. At the beginning of the XXth century, women's health was incorporated into the major national politics, but yet limited to the maternal and child care. After the creation of the PAISM, the focus was directed to the women's health. The National Program of STD/Aids was them created in order to face the dissemination of the HIV. The politics of condoms distribution promoted a greater female autonomy. The PNAISM came to implement the human rights of women. Issues such as gender, individual vulnerability and couples' education provided a greater visibility and power of speech to the marginalized categories.

Descriptors: Public policies; Prostitution; Policy making.

\section{Políticas públicas de la salud dirigidas a la atención a la prostituta: breve rescate histórico}

Estudio reflexivo referente al público de la salud de la mujer, política relativa del notadamente a las prostitutes. Al principio del siglo XX, la salud de la mujer fue incorporada a las políticas nacionales, limitándose a la atención maternal-infantil. Con la creación del PAISM, el foco fue dirigido a la salud de la mujer. El programa nacional de DST/Sida fue creado debido a la difusión del VIH. La política de condones promovió una mayor autonomía femenina. EI PNAISM surgió para poner los derechos humanos de las mujeres en ejecución. Las cuestiones del género, vulnerabilidad individual, educación a los pares proporcionaron una mayor visibilidad y energía de la voz a las categorías marginadas.

Descriptores: Políticas públicas; Prostitución; Formulación de políticas.

\section{INTRODUÇÃO}

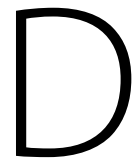

urante muitos anos, regras impostas pela sociedade delegavam à mulher as funções de reprodução e de cuidadoras do lar. A mulher não exercia plenamente sua sexualidade, visto que os limites e a ordem social deveriam ser respeitados. No lar, a relação sexual era mantida dentro dos padrões tradicionais ${ }^{(1)}$.

A relação sexo e reprodução foi culturalmente transmitida ao longo dos anos; apesar disso, a prostituição é uma das profissões mais antigas do mundo. Já no século VI a.C a indústria da prostituição estava organizada, arregimentando escravas para os prostíbulos e criando altos impostos para a prostituição independente ${ }^{(2)}$.

A prostituição pode ser vista como uma forma de resistência, pois a mulher passou a ocupar um espaço até então exclusivo dos homens, deixando o ambiente domiciliar para frequentar as ruas (3). Na Idade Média, consolidou-se o desprezo pelo corpo feminino e, as prostitutas, portadoras do prazer sexual, passaram a ser agente do demônio, o que repercutiu no estigma contra a prostituição vigente até os dias de hoje ${ }^{(2)}$.

Fugir a regras pré-estabelecidas por padrões culturais gera conflitos e traz consequências, muitas vezes, estigmatizantes e segregadoras para os desviantes dessas normas. Não se pode deixar de considerar os preconceitos socialmente constituídos por uma cultura que adotou o modelo da família nuclear burguesa como referência para o padrão sexual, aliando-se com as demandas da Revolução Industrial e com a dominação e subordinação decorrentes 
do capitalismo ${ }^{(4)}$.

Devido à situação de miséria da grande massa populacional, as mulheres passaram a ocupar as ruas em busca de melhores condições de vida. Começaram também a contribuir na renda familiar. Contudo, a escassa formação, falta de experiência profissional e de oportunidades, fizeram com que exercessem funções menos valorizadas e com menor remuneração, instigando-as a buscar meios mais lucrativos, entre eles a prostituição.

A prostituição pode ser definida como a comercialização da prática sexual, geralmente em troca de favores ou vantagens monetárias $^{(5)}$. Considera-se que foi durante a Revolução Industrial que houve um aumento da prostituição em toda a Europa, devido, principalmente, à imigração, às situações de extrema pobreza e à promiscuidade decorrentes das aglomerações urbanas ${ }^{(6)}$.

A origem do estigma e do preconceito existente ainda hoje na sociedade deriva tanto da transgressão das normas e padrões de conduta, quanto da correlação entre prostitutas e doenças sexualmente transmissíveis, presente nas primeiras campanhas de prevenção. Desse modo, a prostituição situava-se no campo das atividades remuneradas ilegítimas e como tais, foi incorporada à reflexão médica como uma doença que ameaçava a população e deveria ser controlada (7).

Durante os primeiros anos da história do HIV/Aids, o conceito de grupo de risco era fortemente atrelado aos homo/ bissexuais, além dos profissionais de sexo. Entende-se por profissionais do sexo, homens e mulheres que comercializam a prática sexual, conhecidos como prostitutas, travestis e michês. As más condições de trabalho, representadas por ausência de práticas sexuais seguras devido à dificuldade de aquisição de preservativos, torna essa população potencial alvo para aquisição de DST/Aids (8).

As prostitutas sempre estiveram inseridas nas campanhas da Saúde Pública, vinculadas às ações preconceituosas, que associavam suas práticas ao risco das DST/Aids. Esse preconceito em relação às prostitutas, presente em nossa sociedade desde tempos remotos, interfere no atendimento dos profissionais de saúde em sua prática. Por medo ou vergonha, as prostitutas acabam por desistir de comparecer ao serviço de saúde para usufruir de seus direitos como mulher. Isso porque os rótulos ainda estão presentes na rede de atenção básica.

A partir da década de 1980 surgiram movimentos de prostitutas em todo o mundo, buscando uma maior articulação entre as mesmas. Em 1986, por ocasião da realização de um Congresso no Rio de Janeiro, surgiu a Rede Brasileira de Prostitutas, que hoje é formada por 25 associações. Esta objetiva promover um maior envolvimento político das prostitutas e o fortalecimento da identidade profissional da categoria, buscando o exercício pleno da cidadania, a redução do estigma e da discriminação, além da melhoria da qualidade de vida na sociedade.

A partir de 2002, a prostituição passou a ser reconhecida pelo Ministério do Trabalho, na categoria de trabalhos informais. Porém, os direitos trabalhistas não foram garantidos, sendo uma importante reivindicação do movimento atual ${ }^{(3)}$.

No Ceará, a Associação das Prostitutas do Ceará (APROCE), criada em 1990, desenvolve trabalho educativo sobre sexualidade, prevenção de DST/Aids, distribuição de preservativos, além de incentivar a busca pelos serviços de saúde para mulheres prostitutas e adolescentes. $\mathrm{O}$ trabalho é realizado pelas educadoras sociais, prostitutas e exprostitutas que se tornaram multiplicadoras de saúde.

Diante do contexto social e político, no qual as prostitutas estão inseridas, questionamos o modo de inclusão das prostitutas nas políticas públicas de saúde, sua participação na elaboração e execução das mesmas, além da sucessão de novas políticas visando englobar as diversidades da população feminina.

Assim, objetivamos no presente estudo realizar um resgate histórico das políticas publicas que contemplam a saúde da mulher, notadamente as relativas às prostitutas, refletindo sobre avanços obtidos por este grupo populacional.

\section{As políticas públicas de saúde voltadas à atenção à mulher com ênfase nas prostitutas}

No Brasil, desde as primeiras décadas do século $X X$, a saúde da mulher foi incorporada às políticas nacionais de saúde, mas os programas se limitavam à atenção materno-infantil, com ênfase no pré-natal e controle de partos ${ }^{(9)}$. A criação do PAISM (Programa de Atenção Integral à Saúde da Mulher), em 1984, surgiu com a constatação da limitação do cuidado da saúde da mulher ${ }^{(10)}$.

O PAISM é considerado um marco histórico, pois orientou um novo enfoque nas políticas públicas voltadas para a Saúde da Mulher e Saúde Reprodutiva, priorizando um atendimento integral e equitativo, envolvendo a saúde da mulher em todas as fases de seu ciclo vital ${ }^{(9)}$.

O Programa almejava, em suas linhas de ação, um modelo assistencial abrangendo a integralidade e a equidade da saúde da mulher. As atividades consistiam em ações educativas, de prevenção, diagnósticas, de tratamento e/ ou recuperação, que objetivavam a melhoria da saúde da população feminina (11).

As ações envolviam uma atenção integral, clínicoginecológica e educativa, que aperfeiçoasse o prénatal, o parto e o puerpério, controle das DST, do câncer cérvico-uterino e mamário e assistência para concepção e contracepção ${ }^{(10)}$.

Vale ressaltar que, quando se discutiu saúde da mulher nas conferências internacionais do Cairo e Beijing, o processo já se 
encontrava bastante avançado conceitualmente no Brasil (12).

O Movimento Feminista vem acompanhando o Programa desde sua elaboração, sendo o mesmo fruto do debate sobre controle de fecundidade e natalidade ${ }^{(13)}$. As intensas políticas de distribuição de pílulas anticoncepcionais e a realização de laqueaduras tubárias fizeram com que as feministas desconfiassem que se tratasse de um programa de controle de natalidade, devido à exclusão do homem no planejamento. Apesar disso, ao longo dos anos, o PAISM revelou-se um avanço na assistência à mulher, contemplando questões outrora esquecidas.

A criação do Programa Nacional de DST/Aids, em 1985, veio confirmar a priorização de estratégias relacionadas à área de saúde sexual e reprodutiva no Brasil, principalmente no tocante à disseminação do HIV.

Os homossexuais e, posteriormente, as prostitutas foram considerados a causa do início da epidemia. Porém, esse fato torna-se compreensível, considerando-se a constituição de uma sociedade machista e moralista. No caso de mulheres que transgridem as normas morais e sociais de conduta, todo o ônus recai sobre elas ${ }^{(14)}$.

Nesse período, surgiu o conceito de grupo de risco, envolvendo atores sociais marginalizados, com foco de ações a esse grupo. A partir de 1994, com o apoio técnico e financeiro para projetos de controle das DST/Aids, houve uma melhor estruturação das redes sociais nos trabalhos de prevenção e assistência, resultando numa resposta mais adequada ao impacto social da epidemia ${ }^{(15)}$.

Atualmente, o termo "grupo de risco" entrou em desuso, sendo utilizada a expressão comportamento de risco, com seu significado relacionado a práticas sexuais que oferecem mais risco de infecção, independente da orientação sexual ${ }^{(16)}$

A prevenção e o controle da transmissão das DST/HIV têm como principal estratégia a realização de atividades educativas que priorizem mudanças no comportamento sexual, percepção do risco, além da oferta de informações que garanta a adoção de medidas preventivas, com ênfase no uso de preservativo ${ }^{(17)}$.

Sabendo da eficácia do preservativo para prevenção de DST/Aids, grande parte das prostitutas, considerando-se sob maior risco, está se protegendo. O consumo de camisinha e o seu uso "na zona" vêm aumentando de maneira bem mais veloz do que em outros segmentos da sociedade (14).

O novo panorama da doença apresenta a feminização, a pauperização e a interiorização da epidemia. Reconhece-se a necessidade de desenvolvimento de ações junto às mulheres, com diversidade social de gênero, identidade sexual, raça e etnia, estabelecendo estratégias para as diferentes populações femininas (15).

A feminização da Aids se apresentou com um crescimento proporcional significativo de mulheres infectadas, apesar de os homens apresentarem maior número de casos. A partir da década de 1990, a vulnerabilidade de mulheres apresentou visibilidade para as autoridades de saúde ${ }^{(18)}$.

Essa situação de feminização da Aids reflete a não priorização desse segmento nas campanhas de prevenção da década de 1980, que eram voltadas exclusivamente para o sexo masculino e para os homossexuais, além das precárias políticas públicas de saúde destinadas à mulher (8)

As relações de desigualdade, historicamente construídas, que colocavam a mulher em situação de vulnerabilidade, mostraram a necessidade de agregar conceitos elucidativos, como o de gênero às políticas públicas ${ }^{(15)}$.

A prevenção se tornou uma questão política, pois depende da construção de redes de mobilização que permitam as transformações necessárias à redução da vulnerabilidade ao HIV/Aids ${ }^{(19)}$. A nova configuração da epidemia revela a existência de um conjunto de fatores que influencia na aquisição do vírus ${ }^{(20)}$.

A vulnerabilidade envolve desde a baixa autoestima, falta de informação sobre os riscos à saúde e sentimento de exclusão, até aspectos relacionados à inserção na sociedade e ao desenvolvimento de ações institucionais voltadas para a Aids como informação, educação, saúde e nãodiscriminação ${ }^{(19)}$.

Uma das estratégias de prevenção das DST/Aids em mulheres, relaciona-se à capacidade de lideranças femininas, dentre estas, prostitutas, como multiplicadoras de saúde. Além disso, garantir a distribuição de preservativos femininos nos serviços de saúde e preservativos masculinos e femininos junto a populações específicas facilita a negociação do uso, autoestima e dupla proteção ${ }^{(15)}$.

Os elevados índices de DST e sua relação com o HIV justificam a inclusão da atenção às DST/Aids nas ações de Saúde Reprodutiva, por ocorrer majoritariamente em pessoas em idade fértil ${ }^{(17)}$. No Brasil, estima-se que $600 \mathrm{mil}$ pessoas sejam portadoras do HIV e que 30 milhões estejam infectadas por alguma DST ${ }^{(19)}$.

A partir de 2001 surgiu a Política de Preservativos Masculinos e Femininos do Ministério da Saúde, como parte integrante do Programa Nacional de DST/Aids. Em 2003 foram distribuídos 256,7 milhões de preservativos masculinos e 2,5 milhões de preservativos femininos (9). Essa distribuição alcançou populações vulneráveis como usuários de drogas e prostitutas.

Diversas ações têm sido realizadas junto a prostitutas, propiciando visibilidade e poder de voz. Em 2002, ocorreu - Seminário Nacional Aids e Prostituição, em Brasília, convocado e patrocinado pelo Ministério da Saúde. O mesmo objetivou discutir as políticas de prevenção das DST/ Aids para essa população, e buscar estratégias de incorporação governamental nessa área. Uma das principais 
reivindicações estava relacionada à disponibilização do preservativo feminino como forma de promover a autonomia feminina, dispensando a argumentação e o consentimento do parceiro. Muitas vezes, a utilização de métodos de barreira é inegociável para o cliente, que chega a oferecer mais pela prática desprotegida.

Mesmo com o incentivo à proteção do HIV/Aids e estando de acordo com ela, as prostitutas não se encontram efetivamente equipadas para negociar o sexo seguro nos programas com clientes resistentes ${ }^{(14)}$.

Neste Seminário foi criado um documento que abordou a preparação dos serviços para o atendimento e acolhimento integrais. Além disso, sugeriu que a instituição de horários e agendamentos adaptados às necessidades das prostitutas, das trabalhadoras e dos usuários de drogas permitiriam melhor inserção dos mesmos nos serviços de saúde. Porém, este documento fez referência à discriminação positiva, conhecida como o ato de privilegiar determinadas classes que outrora foram marginalizadas pela sociedade, pois reconheceu que as dificuldades de acesso à assistência no SUS são as mesmas do restante da população. Houve, ainda, menção à promoção de ações de redução de danos ao uso de drogas, sugerindo a continuidade das ações, dada a contradição entre a cobertura das ações e o tamanho da população.

Outras reivindicações se referem ao incentivo da organização social de profissionais do sexo, com atividades de sensibilização dos profissionais de saúde dos serviços para que realizem uma prática alheia a qualquer tipo de discriminação. Porém, para que isso ocorresse seria necessário abdicar dos preconceitos adquiridos ao longo dos anos.

Pode-se definir preconceito como conceito ou opinião formados antes de ter os conhecimentos adequados (21). Desse modo, para que as ações de promoção da saúde sejam eficazes, deve-se tratar de forma igualitária todos que buscam saúde.

Uma importante ênfase é dada na formulação de políticas públicas que garantam os direitos humanos com controle das classes sociais, assegurando a sustentabilidade das ações de prevenção e assistência realizadas.

A Política de Redução de Danos, elaborada a partir de 1994 é uma estratégia de Saúde Pública que visa reduzir os danos à saúde em consequência de práticas de risco. São realizadas ações de prevenção de danos, primordialmente a pessoas de risco, que oportunizam maior visibilidade a populações historicamente excluídas do SUS.

Considera-se necessário incorporar estratégias de redução de danos nos serviços de assistência à saúde, objetivando promover o aumento da cobertura das demandas e sua adaptação a populações específicas. Como visa a incorporação de práticas seguras, estima-se que a adoção de medidas de prevenção das DST/Aids são complexas e dependentes de situação social, personalidade e consumo de outras substâncias (22).

A partir de 2003, com a elaboração da Política Nacional de Atenção Integral à Saúde da Mulher (PNAISM), o Governo Federal refletiu o compromisso de implementar políticas públicas que garantissem os direitos humanos das mulheres. O programa abrangeu os princípios e a filosofia do PAISM, além de um plano de ação, considerando questões de gênero, raça e etnia. O PNAISM contemplou grupos sociais historicamente excluídos das políticas. Além disso, priorizou ações de redução da mortalidade materna e neonatal e os direitos sexuais e reprodutivos ${ }^{(23)}$.

Essa nova política abordou as inúmeras especificidades da mulher, reconhecendo a necessidade de novas abordagens em distintos grupos: lésbicas, negras, em situação de presídio, trabalhadoras rurais e mulheres indígenas. Fragmentou a mulher em suas diversidades, reorientando a política de saúde para as necessidades de cada grupo populacional.

A fragmentação oriunda dessa nova proposta não se fundamentou em suscetibilidade às doenças e sim à dificuldade de acesso aos serviços de saúde, seja por horário de funcionamento ou por conscientização. Levou em consideração questões inerentes a esses grupos ao longo dos anos. Porém, com tamanha estratificação da população feminina, há um grande risco de as ações de saúde da mulher ficarem dispersas e perderem o foco principal.

Considerando-se as dificuldades para usufruir dos benefícios que o SUS disponibiliza, faz-se necessário que se desenvolvam meios e estratégias a fim de alcançar as prostitutas, que apresentam suscetibilidade à aquisição de doenças sexualmente transmissíveis, à violência física e à discriminação de classe.

\section{Considerações finais}

Diante do exposto, observamos que, ao longo dos anos, populações específicas ficaram à margem da sociedade, excluídas de políticas públicas sociais que garantissem a execução dos direitos humanos universais. Neste breve resgate, pudemos acompanhar as políticas públicas de saúde voltadas para a saúde da mulher, às quais procuram atender a condições específicas de cada momento histórico. Na medida em que o SUS se estrutura e se implementa na prática cotidiana, busca-se seu aprimoramento e proximidade com a realidade.

As necessidades próprias de cada grupo social passam a ser melhor contempladas e atendidas à medida que isso se torna fonte de inquietação desse e/ou de outros segmentos sociais, fazendo contraponto com outras demandas na construção de políticas públicas includentes. Desse modo, quando as prostitutas se constituem como categoria social 
organizada, que busca reconhecimento, aceitação e respeito, ampliam-se seus espaços de atuação e de influência na tomada de decisões políticas, conquistando para a classe direitos antes restritos.

As prostitutas, alvos potenciais das primeiras campanhas higienistas que combatiam a epidemia das doenças venéreas, foram, ao longo dos anos, inserindo-se no contexto da execução de políticas que tentavam favorecer a situação de saúde da população em geral.

Aspectos importantes relacionados à prevenção das DST/Aids, enfocando questões de gênero, vulnerabilidade individual e não mais grupos de risco, educação aos pares, incentivando à organização social, entre outras coisas, proporcionaram maior visibilidade e poder de voz às categorias marginalizadas. Grupos específicos colaboram e participam da formulação, implementação e avaliação das políticas de saúde. Além disso, a política de redução de danos conseguiu alcançar usuários de drogas e prostitutas, com a distribuição maciça de preservativos e seringas.

A atual política de saúde voltada para a mulher (PNAISM), fragmenta o ser mulher nas suas diversas peculiaridades, reorientando as ações de saúde para as especificidades de cada grupo. Porém, certas categorias da população feminina não foram contempladas com ações específicas, dentre elas as prostitutas.

A nova abordagem na assistência à saúde objetiva contemplar o cliente de forma integral. A ausência de critérios de saúde específicos que embasem essa abordagem fragmentada, além do argumento do direcionamento das ações, confronta com os pressupostos e diretrizes do Sistema Único de Saúde (SUS).

Sendoassim, éimportantequeos profissionais de saúdeestejam familiarizados com as políticas de saúde vigentes, suas principais diretrizes norteadoras, para que promovam uma atenção voltada para as necessidades da população, desenvolvendo ações inclusivas e que respondam aos preceitos do SUS.

\section{Referências}

\author{
1. Mazzieiro JB. Sexualidade criminalizada: prostituição, lenocínio e outros \\ delitos. Rev Bras Hist. 1998;18(35):75-87. \\ 2. São Paulo (Estado). Cidade do conhecimento. Corpo e história. Ciência, saber, \\ poder e prazer [lnternet]. [citado 2006 Nov 02]. Disponível em: http://www.cidade. \\ usp.br/educar/?monografias/masculinofeminino/corpohist1/6 \\ 3. Rodrigues A. Sem máscara: prostituta quer profissão regulamentada [Internet]. \\ [citado em 2009 Nov 10]. \\ Disponivel em: http://www.carb.ufba.br/artigos/semmascara-anai.html \\ 4. Ferreira MPS. Aids: da violência aos direitos humanos à construção da \\ solidariedade [dissertação]. Rio de Janeiro: Universidade Federal do Rio de \\ Janeiro;1999. \\ 5. Wikipedia. Enciclopédia livre [Internet]. [citado em 2009 Dez 8]. Disponível em: \\ http://pt.wikipedia.org/wiki/Prostitui\%C3\%A7\%C3\%A30 \\ 6. Passos ADC, Figueiredo JFC. Fatores de risco para doenças sexualmente \\ transmissiveis entre prostitutas e travestis de Ribeirão Preto (SP), Brasil. \\ Rev Panam Salud Publica [Internet]. [citado 2007 Jun 16]. \\ Disponivel em: http://www.scielosp.org/pdf/rpsp/v16n2/22243.pdf 16(2), 2004 \\ 7. Gomes R. Prostituição infantil: uma questão de saúde pública. \\ Cad Saúde Públ. 1994;10(1):58-66.
}

8. Schaurich D, Padoin SMM. Do cuidado da mulher: questões de gênero e sua incorporação no contexto do HIV/Aids. Esc Anna Nery R Enferm. 2004;8(1):101-8. 9. Brasil. Ministério da Saúde. Secretaria de Atenção à Saúde. Direitos sexuais e direitos reprodutivos - uma prioridade do governo. Brasilia: Ministério da Saúde; 2005. 10. Osis MJMD. Paism: um marco na abordagem da saúde reprodutiva no Brasil. Cad Saúde Públ. 1998;(1 Suppl).

11. Formiga Filho JFN. Políticas de Saúde Reprodutiva no Brasil: uma análise do PAISM. In: Galvão L, Diaz J, (organizadores). Saúde sexual e reprodutiva no Brasil: dilemas e desafios. São Paulo: Hucitec Population Council; 1999. p. 151-62. 12. Díaz M, Díaz J. Qualidade de atenção em Saúde Sexual e Reprodutiva: estratégias para mudanças. In: Galvão L, Diaz J, (organizadores).

Saúde sexual e reprodutiva no Brasil: dilemas e desafios. São Paulo: Hucitec

Population Council; 1999. p. 151-62.

13. Oliveira F. Saúde integral para as mulheres: ontem, hoje e perspectivas. J Rede Fem Saúde. 2005;5(27):6-9.

14. Brasil. Ministério da Saúde. Secretaria de Assistência à Saúde.

Manual do multiplicador. Profissionais do sexo. Brasilia: Ministério da Saúde; 1996.

15. Brasil. Ministério da Saúde. Coordenação de DST/Aids. Políticas e diretrizes

de prevenção das DST/Aids entre mulheres. Brasilia: Ministério da Saúde; 2003.

16. Brasil. Ministério da Saúde. Secretaria de Políticas de Saúde. Guia de prevenção

das DST/Aids e cidadania para homossexuais. Brasília: Ministério da Saúde; 2002.

17. Ceará. Secretaria de Saúde. Saúde reprodutiva e sexual: um manual para a

atenção primária e secundária (nível ambulatorial). Fortaleza: SESA-CE; 2002.

18. Martins TA. Doenças sexualmente transmissíveis em mulheres grávidas:

um estudo sobre prevalência e fatores de risco [dissertação].

Fortaleza: Universidade Federal do Ceará; 2002.

19. Brasil. Ministério da Saúde. Centro de Referência e treinamento DST/Aids. Projetos estratégicos com populaçōes vulneráveis.

São Paulo: Ministério da Saúde; 2005.

20. Mann CG, Oliveira SB, Oliveira CSS. Guia para profissionais de saúde

mental - sexualidade e DST/Aids: discutindo o subjetivo de forma objetiva.

Rio de Janeiro: Instituto Franco Basaglia; 2002.

21. Dicionário Língua Portuguesa. São Paulo: Melhoramentos; 2002.

22. Brasil. Ministério da Saúde. Secretaria de Vigilância a Saúde.

Política de redução de danos [Internet]. [citado em 2006 Nov 26].

Disponível em: http://portal.saude.gov.br/portal/aplicacoes/noticias/

noticias_detalhe.cfm?co_seq_noticia $=5693$

23. Araújo MJO. Política Nacional de Atenção Integral à Saúde da Mulher: antigas necessidades e novas perspectivas. J Rede Fem Saúde. 2005;5(27):28-30. 